\title{
Using GH-Method: Math-Physical Medicine to Conduct Segmentation Analysis to Investigate the Impact of both Weight and Weather Temperatures on Fasting Plasma Glucose (FPG)
}

\author{
Gerald C. Hsu* \\ Medical Research Scientist, EclaireMD Fondation, 7 Oak Haven Way, Woodside, CA 94062 USA. \\ *Corresponding Author: Gerald C. Hsu, Medical Research Scientist, EclaireMD Fondation, 7 Oak \\ Haven Way, Woodside, CA 94062 USA.
}

\section{INTRODUCTION}

This paper is based on a big data collected from a period of 1,420 days (from $6 / 1 / 2015$ to $4 / 21 / 2019)$ with a total of 4,260 data, including morning weight in pounds (lbs.), highest ambient temperature (weather) of each day in degree Fahrenheit $\left({ }^{\circ} \mathrm{F}\right)$ and fasting plasma glucose or FPG $(\mathrm{mg} / \mathrm{dL})$. The dataset is provided by the author, who uses his own type 2 diabetes metabolic conditions control, as a case study via the "math-physical medicine" approach of a non-traditional methodology in medical research.

Math-physical medicine (MPM) starts with the observation of the human body's physical phenomena (not biological or chemical characteristics), collecting elements of the disease related data (preferring big data), utilizing applicable engineering modeling techniques, developing appropriate mathematical equations (not just statistical analysis), and finally predicting the direction of the development and control mechanism of the disease.

\section{METHOD}

In this analysis, the author defines his ideal weight at $170 \mathrm{lbs}$. (BMI 25) and the following three weather temperature ranges:

(1) Chilly: $<67^{\circ} \mathrm{F}$

(2) Comfortable: $67-77^{\circ} \mathrm{F}$

(3) Warm: $>77^{\circ} \mathrm{F}$

The author used the GH-Method: math-physical medicine (MPM) approach to discover the impact on FPG by both weight and weather temperature in 2016. His published papers have indicated that weight contributes $\sim 85 \%$ of FPG formation and temperature contributes $\sim 10 \%$. Currently, he conducted a detailed segmentation analysis to further validate his earlier findings.

\section{RESULTS}

Based on the chilly temperature range (average: $59^{\circ} \mathrm{F}$ ) and normal weight condition (average: 169.8 lbs.), he achieved an average FPG at 99 $\mathrm{mg} / \mathrm{dL}$.

The similarity of data patterns and FPG impact distinction between primary factor (weight) and secondary factor (temperature) can also be visually observed from three graphs in Figure 2. 
Using GH-Method: Math-Physical Medicine to Conduct Segmentation Analysis to Investigate the Impact of both Weight and Weather Temperatures on Fasting Plasma Glucose (FPG)

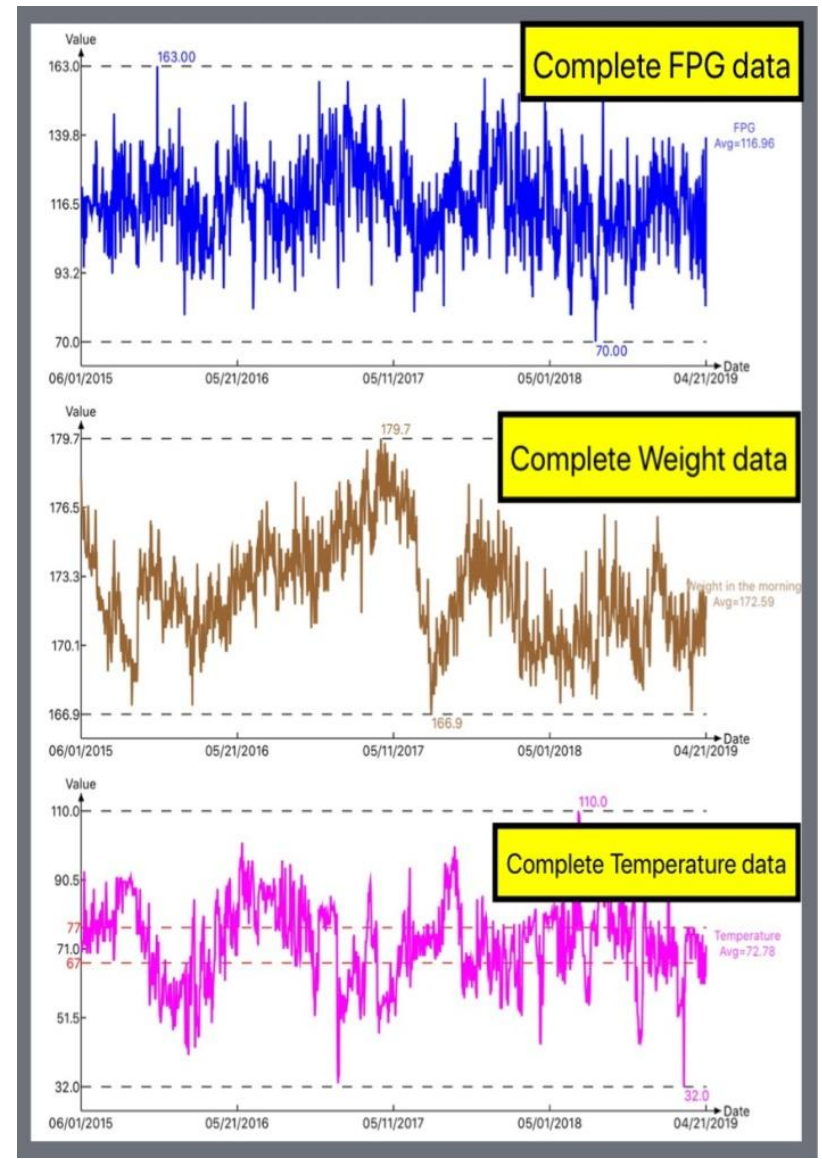

Figure 1: Complete datasets of FPG, Weight, \& Weather Temperature

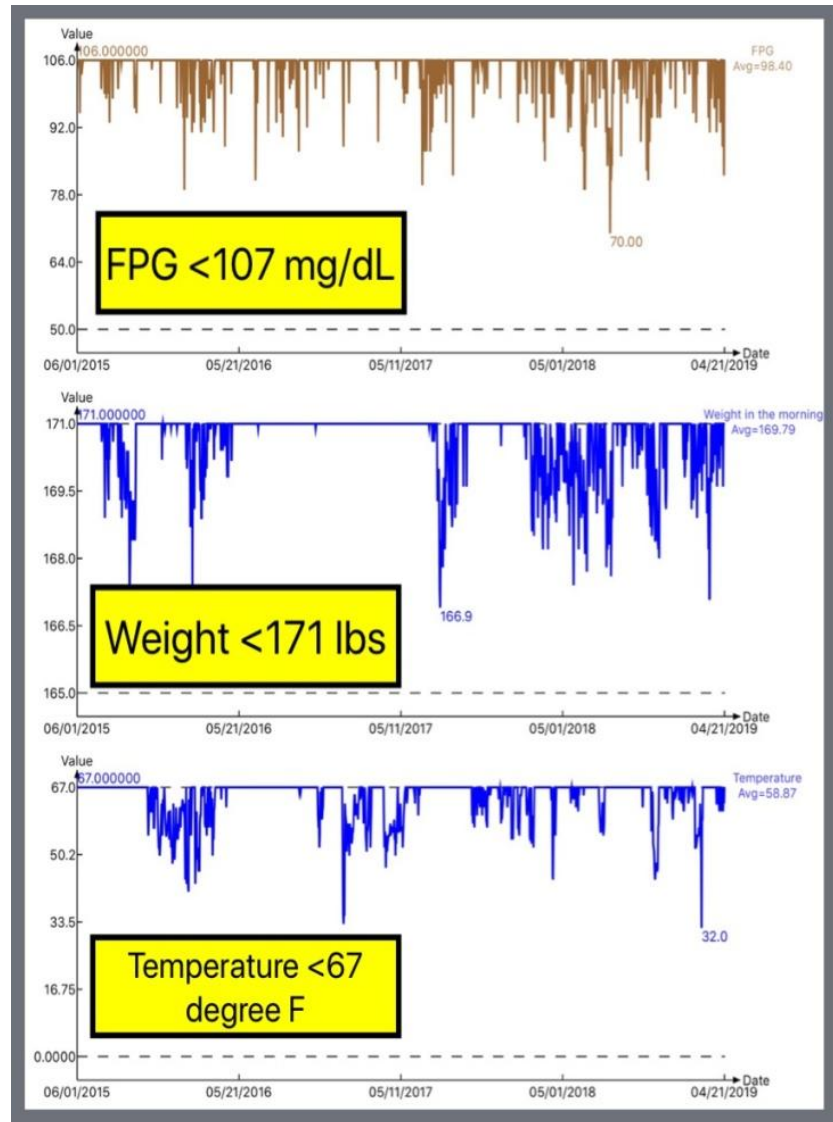

Figure 2: Data for Chilly weather temperature $\left(<67-\right.$ degree $\left.^{\circ} \mathrm{F}\right)$, normal weight $(<171 \mathrm{lbs}$.$) , and lower FPG$ $(<107 \mathrm{mg} / \mathrm{dL})$ 
Using GH-Method: Math-Physical Medicine to Conduct Segmentation Analysis to Investigate the Impact of both Weight and Weather Temperatures on Fasting Plasma Glucose (FPG)

\section{CONCLUSION}

By using the GH-Method: math-physical medicine on this big data, the author proved again that the correlation of weight/temperature and FPG by this segmentation analysis. The important role of weight on FPG has been demonstrated repeated via different clinical cases. However, due to unknown biological reactions of human body and unavailability of applicable data from individuals who reside in either tropical or freezing zones, the author cannot draw the same conclusions of temperature impact on FPG formation.

\section{REFERENCES}

[1] Hsu, Gerald C. (2018). Using Math-Physical Medicine to Control T2D via Metabolism Monitoring and Glucose Predictions. Journal of Endocrinology and Diabetes, 1(1), 1-6.
[2] Hsu, Gerald C. (2018). Using Signal Processing Techniques to Predict PPG for T2D. International Journal of Diabetes \& Metabolic Disorders, 3(2), 1-3.

[3] Hsu, Gerald C. (2018). Using Math-Physical Medicine and Artificial Intelligence Technology to Manage Lifestyle and Control Metabolic Conditions of T2D. International Journal of Diabetes \& Its Complications, 2(3), 1-7.

[4] Hsu, Gerald C. (2018). Using Math-Physical Medicine to Study the Risk Probability of having a Heart Attack or Stroke Based on Three Approaches, Medical Conditions, Lifestyle Management Details, and Metabolic Index. EC Cardiology, 5(12), 1-9.

Citation: Gerald C. Hsu, "Using GH-Method: Math-Physical Medicine to Conduct Segmentation Analysis to Investigate the Impact of both Weight and Weather Temperatures on Fasting Plasma Glucose (FPG)", International Journal of Research Studies in Medical and Health Sciences. 2020; 5(5): 03-05.

Copyright: (c) 2020 Gerald C. Hsu, This is an open-access article distributed under the terms of the Creative Commons Attribution License, which permits unrestricted use, distribution, and reproduction in any medium, provided the original author and source are credited. 